\title{
rs1990622 variant associates with Alzheimer's disease and regulates TMEM106B expression in human brain tissues
}

Yang Hư ${ }^{1}$, Jing-yi Sun ${ }^{2}$, Yan Zhang ${ }^{3}$, Haihua Zhang ${ }^{4}$, Shan Gao ${ }^{4}$, Tao Wang ${ }^{5,6}$, Zhifa Han ${ }^{7,8,9}$, Longcai Wang ${ }^{10}$, Bao-liang Sun ${ }^{11}$ and Guiyou Liu $4,6,11,12,13^{*}$

\begin{abstract}
Background: It has been well established that the TMEM106B gene rs 1990622 variant was a frontotemporal dementia (FTD) risk factor. Until recently, growing evidence highlights the role of TMEM106B in Alzheimer's disease (AD). However, it remains largely unclear about the role of rs1990622 variant in AD.

Methods: Here, we conducted comprehensive analyses including genetic association study, gene expression analysis, eQTLs analysis, and colocalization analysis. In stage 1, we conducted a genetic association analysis of rs 1990622 using large-scale genome-wide association study (GWAS) datasets from International Genomics of Alzheimer's Project (21,982 AD and 41,944 cognitively normal controls) and UK Biobank (314,278 participants). In stage 2, we performed a gene expression analysis of TMEM106B in 49 different human tissues using the gene expression data in GTEx. In stage 3, we performed an expression quantitative trait loci (eQTLs) analysis using multiple datasets from UKBEC, GTEx, and Mayo RNAseq Study. In stage 4, we performed a colocalization analysis to provide evidence of the AD GWAS and eQTLs pair influencing both AD and the TMEM106B expression at a particular region.

Results: We found (1) rs1990622 variant T allele contributed to AD risk. A sex-specific analysis in UK Biobank further indicated that rs 1990622 T allele only contributed to increased AD risk in females, but not in males; (2) TMEM106B showed different expression in different human brain tissues especially high expression in cerebellum; (3) rs1990622 variant could regulate the expression of TMEM106B in human brain tissues, which vary considerably in different disease statuses, the mean ages at death, the percents of females, and the different descents of the selected donors; (4) colocalization analysis provided suggestive evidence that the same variant contributed to AD risk and TMEM106B expression in cerebellum.

Conclusion: Our comprehensive analyses highlighted the role of FTD rs1990622 variant in AD risk. This cross-disease approach may delineate disease-specific and common features, which will be important for both diagnostic and therapeutic development purposes. Meanwhile, these findings highlight the importance to better understand TMEM106B function and dysfunction in the context of normal aging and neurodegenerative diseases.
\end{abstract}

Keywords: TMEM106B, Alzheimer's disease, Genome-wide association study, Neurological diseases, eQTLs

\footnotetext{
* Correspondence: liuguiyou1981@163.com

${ }^{4}$ Beijing Institute for Brain Disorders, Laboratory of Brain Disorders, Ministry of Science and Technology, Collaborative Innovation Center for Brain Disorders,

Capital Medical University, Beijing 100069, China

${ }^{6}$ Chinese Institute for Brain Research, Beijing, China

Full list of author information is available at the end of the article
}

(c) The Author(s). 2021 Open Access This article is licensed under a Creative Commons Attribution 4.0 International License, which permits use, sharing, adaptation, distribution and reproduction in any medium or format, as long as you give appropriate credit to the original author(s) and the source, provide a link to the Creative Commons licence, and indicate if changes were made. The images or other third party material in this article are included in the article's Creative Commons licence, unless indicated otherwise in a credit line to the material. If material is not included in the article's Creative Commons licence and your intended use is not permitted by statutory regulation or exceeds the permitted use, you will need to obtain permission directly from the copyright holder. To view a copy of this licence, visit http://creativecommons.org/licenses/by/4.0/. The Creative Commons Public Domain Dedication waiver (http://creativecommons.org/publicdomain/zero/1.0/) applies to the data made available in this article, unless otherwise stated in a credit line to the data. 


\section{Background}

TMEM106B is a lysosomal protein and belongs to the TMEM106 family of proteins with relatively unknown function [1, 2]. In 2010, a common TMEM106B genetic variant rs1990622 (T>C) was first identified to be associated with frontotemporal dementia (FTD) risk (OR = 1.64 for $\mathrm{T}$ allele, allele frequency $=0.679$, and $P=1.08 \mathrm{E}$ -11) [3]. Since the identification of rs1990622 as an FTD risk variant, kinds of studies have been conducted to understand the role of this non-coding mutation, which is located downstream $6.9 \mathrm{~kb} \mathrm{3'}$ of TMEM106B [4-6].

$\mathrm{Li}$ and colleagues conducted a cell type quantitative trait loci (cQTL) analysis using 2008 brain samples derived from 1536 unique individuals including 640 AD, 488 cognitively healthy controls, 11 FTD, 75 progressive supranuclear palsy, 28 pathological aging, 189 schizophrenia, 30 bipolar disorders, and 75 individuals with other unknown dementia or no diagnosis information [4]. Interestingly, Li and colleagues identified TMEM106B variant rs1990621 C allele, which is in high linkage disequilibrium with rs1990622 variant $\mathrm{T}$ allele, to be significantly associated with the reduced neuronal proportion [4].

Until recently, Yang and colleagues conducted a module quantitative trait loci (modQTL) analysis to identify genetic variants regulating the average expression of the genes found in the gene co-expression modules [5]. Interestingly, they found rs1990622 variant to show a significant modQTL effect, and highlighted TMEM106B as key aging human brain transcriptome regulator [5]. Meanwhile, Yang and colleagues identified that myelination and lysosomal genes regulated by TMEM106B could connect amyloid- $\beta(\mathrm{A} \beta)$ and TAR DNA-binding protein $43 \mathrm{kDa}$ (TDP-43) [5]. It is known that increased $\mathrm{A} \beta$ is a key Alzheimer's disease (AD) neuropathology. Hence, Yang and colleagues provided important findings about the key pathogenic link between AD and TDP-43 proteinopathy [5]. However, Yang and colleagues did not directly evaluate the association between rs1990622 variant and AD risk. Until now, it remains unclear whether rs1990622 variant is associated with AD risk, although a lack of significant association between rs1990622 variant and AD risk [7]. We think that this may be caused by inadequate sample sizes (300 AD cases and 137 controls) [7], and large-scale samples are needed.

Meanwhile, Yang and colleagues conducted an expression quantitative trait loci (eQTLs) analysis of rs1990622 variant using 494 human prefrontal cortex samples from the Religious Orders Study and Memory and Aging Project (ROSMAP) [8]. They found that rs1990622 variant $\mathrm{T}$ allele could significantly increase TMEM106B expression ( $\beta=0.067$, and $P=5.90 \mathrm{E}-05$ ) [5]. However, gene expression analysis did not support the increased TMEM106B expression in human brain tissues. Satoh et al. evaluated the expression levels of TMEM106B in
$\mathrm{AD}$ and control frontal cortex and the hippocampus tissues [9]. They selected 6 sporadic AD patients and 13 controls including 4 normal subjects without neurological disease, 3 patients with sporadic Parkinson's disease, 4 patients with sporadic amyotrophic lateral sclerosis, and 2 patients with sporadic multiple system atrophy [9]. They demonstrated that both the mRNA and protein levels of TMEM106B were significantly reduced in $\mathrm{AD}$ brains compared control brains [9].

In discussion, Yang and colleagues concluded that the pre-existing neurodegenerative proteinopathies were not necessary to drive the association between rs1990622 variant and TMEM106B transcriptome dysregulation [5]. However, recent findings from other TMEM106B variants did not support this conclusion. Ren and colleagues conducted a stratification analysis and highlighted more pronounced effects of TMEM106B rs3173615 variant on the transcriptome in neurodegenerative diseases than in healthy controls [6]. Li and colleagues conducted a stratification analysis and found that TMEM106B rs1990621 variant could regulate the neuronal proportion in AD cases, other neurodegenerative diseases, elderly cognitively healthy controls, but not young controls [4]. All these findings indicated that the link between TMEM106B haplotype and transcriptome dysregulation is context dependent $[4,6]$. Importantly, rs1990622 variant is in high linkage disequilibrium with rs3173615 $\left(r^{2}=0.98\right.$ and $\left.D^{\prime}=1\right)$ and $\operatorname{rs} 1990621\left(r^{2}=0.99\right.$ and $D^{\prime}=$ 1). Hence, we consider that the association between rs1990622 variant and TMEM106B transcriptome dysregulation may also be context dependent.

Until now, large-scale AD genome-wide association study (GWAS) datasets and large-scale eQTLs datasets in both the neuropathologically normal individuals and neurological disease individuals have provided strong support to answer these concerns $[10,11]$. Here, we conducted comprehensive analyses using publicly available datasets. In stage 1, we conducted a genetic association analysis to investigate the effect of rs1990622 variant on $\mathrm{AD}$ risk using multiple large-scale GWAS datasets. In stage 2, we performed a gene expression analysis of TMEM106B in 49 different human tissues. In stage 3, we performed an eQTLs analysis to evaluate the effect of rs1990622 variant on TMEM106B expression in multiple human brain tissues with different disease statuses. In stage 4, we performed a colocalization analysis to provide evidence of the AD GWAS and eQTLs pair influencing both $\mathrm{AD}$ and the TMEM106B expression at a particular region.

\section{Methods \\ AD GWAS datasets}

We selected two independent large-scale AD GWAS dataset resources from International Genomics of 
Alzheimer's Project (IGAP) stage 1 [10] and UK Biobank [11]. The IGAP stage 1 consisted of 21,982 AD and 41, 944 cognitively normal controls of European descent [10]. These individuals are from four consortia including Alzheimer Disease Genetics Consortium (ADGC), Cohorts for Heart and Aging Research in Genomic Epidemiology Consortium (CHARGE), the European Alzheimer's Disease Initiative (EADI), and Genetic and Environmental Risk in AD/Defining Genetic, Polygenic and Environmental Risk for Alzheimer's Disease Consortium (GERAD/PERADES) [10]. AD patients are diagnosed using the NINCDS-ADRDA criteria or DSM-IV guidelines [10]. In UK Biobank, AD GWAS was conducted in 314,278 participants including 27,696 maternal cases and 14,338 paternal cases [11]. Meanwhile, UK Biobank also included two sex-specific AD GWAS datasets including one AD GWAS dataset in males diagnosed by paternal history of AD (14,338 cases and 245, 941 controls) and one AD GWAS dataset in females diagnosed by maternal history of AD (27,696 cases and 260,980 controls) [11].

\section{eQTLs datasets from neuropathologically normal and disease individuals}

We selected two independent eQTLs dataset resources from the neuropathologically normal individuals. The first resource is from the UK Brain Expression Consortium (UKBEC), which included 134 neuropathologically normal individuals of European descent [12]. The UKBEC consisted of 10 eQTLs datasets in 10 brain regions including cerebellar cortex, frontal cortex, hippocampus medulla, occipital cortex, putamen, substantia nigra, temporal cortex, thalamus, and intralobular white matter [12]. The second resource is from the Genotype-Tissue Expression (GTEx, version 8) [13]. The GTEx included 13 eQTLs datasets in 13 brain tissues (amygdala/amygdalae, anterior cingulate cortex, caudate basal ganglia, cerebellar hemisphere, cerebellum, cortex, frontal cortex, hippocampus, hypothalamus, nucleus accumbens, putamen, spinal cord, and substantia nigra) [13]. About $99 \%$ of the donors of these brain tissues are neuropathologically normal individuals, and $1 \%$ of the donors of these brain tissues died of neurological diseases (1.3\% in age $20-39$ and $1.2 \%$ in age 60-71) [13]. Recent studies have provided more detailed information about these datasets [14-19].

The eQTLs dataset resource in neurological disease individuals is from the Mayo RNAseq Study [20]. Mayo eQTLs datasets included 773 brain samples, which could be further divided into 197 AD cerebellar samples, 202 AD temporal cortex samples, 177 non-AD cerebellar samples, and 197 non-AD temporal cortex samples [20]. The non-AD samples have several brain pathologies including PSP, LBD, corticobasal degeneration, FTD, multiple system atrophy, and vascular dementia [20].

The main demographic profiles of the selected eQTLs datasets are provided in Table 1. In brief, the selected donors in UKBEC are of European descent with mean age at death 59 and $26 \%$ of these donors were female. The selected donors in GTEx are of multiple descents including European (85.3\%), African (12.3\%), Asian (1.4\%), and Hispanic or Latino (1.9\%), with mean age at death 55, and 33\% of these donors were female. The selected donors in Mayo are of European descent with mean age at death 74 in $\mathrm{AD}$ and 72 in non- $\mathrm{AD}$, and $51-53 \%$ of these donors were female in $\mathrm{AD}$ and $36-40 \%$ of these donors were female in non-AD. In order to further compare the findings from ROSMAP, we also included the demographic profiles of the ROSMAP eQTLs datasets, as described in Table 1. In ROSMAP, the selected donors are of European descent with mean age at death 88 and $62 \%$ of these donors were female. Thirty-nine percent of these donors were diagnosed with clinical $\mathrm{AD}$, and $58 \%$ were pathological $\mathrm{AD}$.

\section{Genetic association analysis}

For the genetic association analysis, we first used the $\mathrm{AD}$ GWAS summary statistics to directly evaluate the

Table 1 Demographic profiles of the selected eQTLs datasets

\begin{tabular}{llllll}
\hline Dataset & Diagnosis & Donors \# & Mean age at death & Descent & Females \% \\
\hline UKBEC & Neuropathologically normal & 134 & 59 & European \\
GTEx & Neuropathologically normal & 838 & 55 & European (85.3\%) & African (12.3\%) \\
& & & & Asian (1.4\%) \\
& & & Hispanic or Latino (1.9\%) & 33 \\
Mayo & AD (cerebellar) & 197 & 74 & European & 51 \\
Mayo & Non-AD (cerebellar) & 177 & 72 & European & 36 \\
Mayo & AD (temporal cortex) & 202 & 74 & European & 53 \\
Mayo & Non-AD (temporal cortex) & 197 & 72 & European & \\
ROSMAP & $39 \%$ clinical AD & 494 & 88 & European & 62 \\
& 58\% pathological AD & & & \\
\hline
\end{tabular}


association of rs1990622 variant with AD in IGAP and UK Biobank. We extract the corresponding summary statistics of rs1990622 variant including the beta coefficient (effect size) and standard error in these datasets, respectively. We then determine the heterogeneity of rs1990622 variant in both datasets using Cochran's $Q$ test [21-26]. Finally, we conducted a meta-analysis to evaluate the association between rs1990622 variant and AD using $\mathrm{R}$ package (meta: General Package for Meta-Analysis) [21-26]. The overall OR is calculated by the fixed effect model (Mantel-Haenszel) or random-effect model (DerSimonian-Laird), which is determined by the heterogeneity [21-26]. Meanwhile, we further perform additional sex stratification analysis only using the UK Biobank GWAS summary datasets. The statistically significant association for heterogeneity test and meta-analysis is defined to be $P<0.05$.

\section{Gene expression analysis of TMEM106B}

In order to evaluate the expression of TMEM106B in different human tissues, we conduct a gene expression analysis using the gene expression data in GTEx (version 8, dbGaP Accession phs000424.v8.p2) [27]. GTEx (version 8) consists of 49 tissues, 838 donors, and 15,201 samples with the number of samples with genotype $\geq 70$ [27]. Illumina TruSeq RNA sequencing and Affymetrix Human Gene 1.1 ST Expression Array were selected to measure the levels of gene expression, which was quantified by transcripts per million (TPM) based on the GENCODE 26 annotation [27]. Here, $T$ test or analysis of variance (ANOVA) was selected to evaluate the potential difference of TMEM106B expression in different human tissues. The statistical significance is $P<0.05$.

\section{eQTLs analysis of rs1990622 variant}

In all these selected eQTLs datasets, an additive model was used to indicate the rs1990622 genotype dosages including $\mathrm{CC}=0, \mathrm{CT}=1$, and $\mathrm{TT}=2$, and a linear regression analysis was used to conduct the eQTLs analysis. For eQTLs analysis in UKBEC, we first downloaded the TMEM106B gene expression data and the rs1990622 genotype data [12]. We then evaluated the association between rs1990622 variant and TMEM106B gene expression using a linear regression analysis [12]. For eQTLs analysis in GTEx (version 8), we used the online GTEx eQTL Calculator with the linear regression method to evaluate the association rs1990622 variant and TMEM106B expression [13]. In Mayo RNAseq Study, a linear regression was used to perform the eQTLs analysis by correcting for APOE $\varepsilon 4$ dosage, age at death, gender, and multiple technical variables [20]. Here, we downloaded the summary results from the Mayo RNAseq Study to directly evaluate the association rs1990622 variant and TMEM106B expression [20]. The statistically significant association is defined to be $P<$ $0.05 / 27=1.85 \mathrm{E}-03$. The suggestive association is defined to be $P<0.05$.

\section{Colocalization analysis}

In order to provide evidence of the AD GWAS and eQTLs pair influencing both $\mathrm{AD}$ and the TMEM106B expression at a particular region, we performed a colocalization analysis using Coloc: a package for colocalization analyses [28, 29]. Coloc could test five hypotheses based on the posterior probability (PP) for colocalization: $\mathrm{HO}-$ neither trait has a genetic association in the region; $\mathrm{H} 1$ / $\mathrm{H} 2$ - only trait 1 /trait 2 has a genetic association in the region; H3-both traits are associated, but with different causal variants; and $\mathrm{H} 4$ - both traits are associated and share a single causal variant $[28,29]$.

\section{Results}

Genetic association analysis of rs 1990622 with $A D$

In IGAP, the results showed that rs1990622 $\mathrm{T}$ allele was significantly associated with increased $\mathrm{AD}$ risk $(P=5.42 \mathrm{E}$ -03). Interestingly, this finding was successfully replicated in UK Biobank $(P=1.20 \mathrm{E}-02)$. Importantly, the sex-specific analysis indicated that rs1990622 $\mathrm{T}$ allele was only significantly associated with increased $A D$ risk in females $(P=5.74 \mathrm{E}-04)$, but not in males $(P=6.48 \mathrm{E}$ -01 ), as provided in Table 2. In both IGAP and UK Biobank, we found no significant heterogeneity with Cochran's $Q$ test $P=0.3833$. A meta-analysis using the fixed effect model showed significant association between rs1990622 variant $\mathrm{T}$ allele and $\mathrm{AD}$ risk $(\mathrm{OR}=$ 1.03, 95\% CI 1.01-1.05, $P=2.00 \mathrm{E}-04)$.

\section{Gene expression analysis of TMEM106B}

In these selected 49 human tissues from GTEx, the top 10 human tissues with high TMEM106B expression are uterus $(n=142$, TPM median $=14.48)$, adrenal gland $(n=258$, TPM median $=13.72)$, cerebellar hemisphere $(n=215$, TPM median $=12.02)$, tibial nerve $(n=619$, TPM median $=11.90)$, cultured fibroblast cells $(n=504$, TPM median $=11.66)$, ovary $(n=180$, TPM median $=$ $11.14)$, tibial artery $(n=663$, TPM median $=10.94)$, thyroid $(n=653$, TPM median $=10.90)$, cerebellum $(n=$

Table 2 Association between rs1990622 variant T allele and AD

\begin{tabular}{lllll}
\hline Dataset & Disease & Beta & SE & $\boldsymbol{P}$ value \\
\hline IGAP & AD & 0.040 & 0.014 & $\mathbf{5 . 4 2 E}-\mathbf{0 3}$ \\
UK Biobank & AD & 0.025 & 0.010 & $\mathbf{1 . 2 0 E}-\mathbf{0 2}$ \\
UK Biobank & AD in females & 0.043 & 0.012 & $\mathbf{5 . 7 4 E}-\mathbf{0 4}$ \\
UK Biobank & AD in males & 0.008 & 0.017 & $6.48 \mathrm{E}-01$
\end{tabular}

$S E$ standard error. Beta is the regression coefficient based on the effect allele. Beta $>0$ and beta $<0$ mean that this effect allele could increase and reduce $A D$ risk, respectively. The statistically significant association is defined to be $P<0.05$ 
241, TPM median $=10.36)$, and spinal cord $(n=159$, TPM median $=10.32$ ). Meanwhile, TMEM106B shows low expression in other 10 human brain tissues including frontal cortex $(n=209$, TPM median $=6.904)$, hypothalamus $(n=202$, TPM median $=6.468)$, nucleus accumbens $(n=246$, TPM median $=5.533)$, caudate $(n=$ 246 , TPM median $=5.286)$, substantia nigra $(n=139$, TPM median $=5.242)$, anterior cingulate cortex $(n=176$, TPM median $=5.237)$, cortex $(n=255$, TPM median $=$ 4.939), hippocampus ( $n=197$, TPM median $=4.848)$, amygdala/amygdalae $(n=197$, TPM median $=4.848)$, and putamen $(n=205$, TPM median $=4.213)$. The box plots about TMEM106B gene expression in different tissues are provided in Fig. 1.

\section{eQTLs analysis}

In UKBEC, eQTLs analysis showed that rs1990622 T allele was not significantly associated with TMEM106B expression in all these 10 brain regions, as provided in Table 3. In GTEx, eQTLs analysis indicated that rs1990622 $\mathrm{T}$ allele was significantly associated with TMEM106B expression in cerebellum $(P=1.90 \mathrm{E}-06)$, cortex $(P=2.20 \mathrm{E}-05)$, and cerebellar hemisphere $(P=$ 1.50E-03). Meanwhile, rs1990622 $\mathrm{T}$ allele also showed suggestive association with TMEM106B expression in frontal cortex $(P=2.20 \mathrm{E}-02)$. Importantly, the rs1990622 $\mathrm{T}$ allele could only significantly reduce TMEM106B expression in these brain tissues, as provided in Table 3. Hence, the eQTLs findings in UKBEC and GTEx from neuropathologically normal individuals did not support the association between rs1990622 $\mathrm{T}$ allele and increased TMEM106B expression even in the same brain tissue, as reported in ROSMAP by Yang and colleagues.

In Mayo datasets, eQTLs analysis indicated no significant association of rs1990622 T allele with TMEM106B expression in cerebellum and temporal cortex (Table 3). Hence, the most significant association between rs1990622 $\mathrm{T}$ allele and reduced TMEM106B expression identified in neuropathologically normal individuals was not successfully replicated in neurological disease individuals. Here, we provided more detailed results about eQTLs analysis in Table 3.

\section{Colocalization analysis}

In GTEx cerebellum eQTLs dataset, we got 181 genetic variants, which could regulate TMEM106B expression with the genome-wide significance level $P<1.00 \mathrm{E}-04$. We then integrated these 181 genetic variants with $A D$ GWAS dataset from IGAP using Coloc. The results showed that TMEM106B had suggestive evidence $(\mathrm{PP} 4=$ $20 \%$ ) of sharing the same variant with $A D$ risk and TMEM106B expression in cerebellum $(\mathrm{PP} 0=0.031$, $\mathrm{PP} 1=0.002$, $\mathrm{PP} 2=0.735, \mathrm{PP} 3=0.037$ ), as described in Fig. 2.

\section{Discussion}

It has been well established that the TMEM106B rs1990622 variant was a FTD risk factor [30, 31]. Until recently, growing evidence highlights the role of TMEM106B in other neurological processes including hippocampal sclerosis of aging [32], neuronal loss [31],

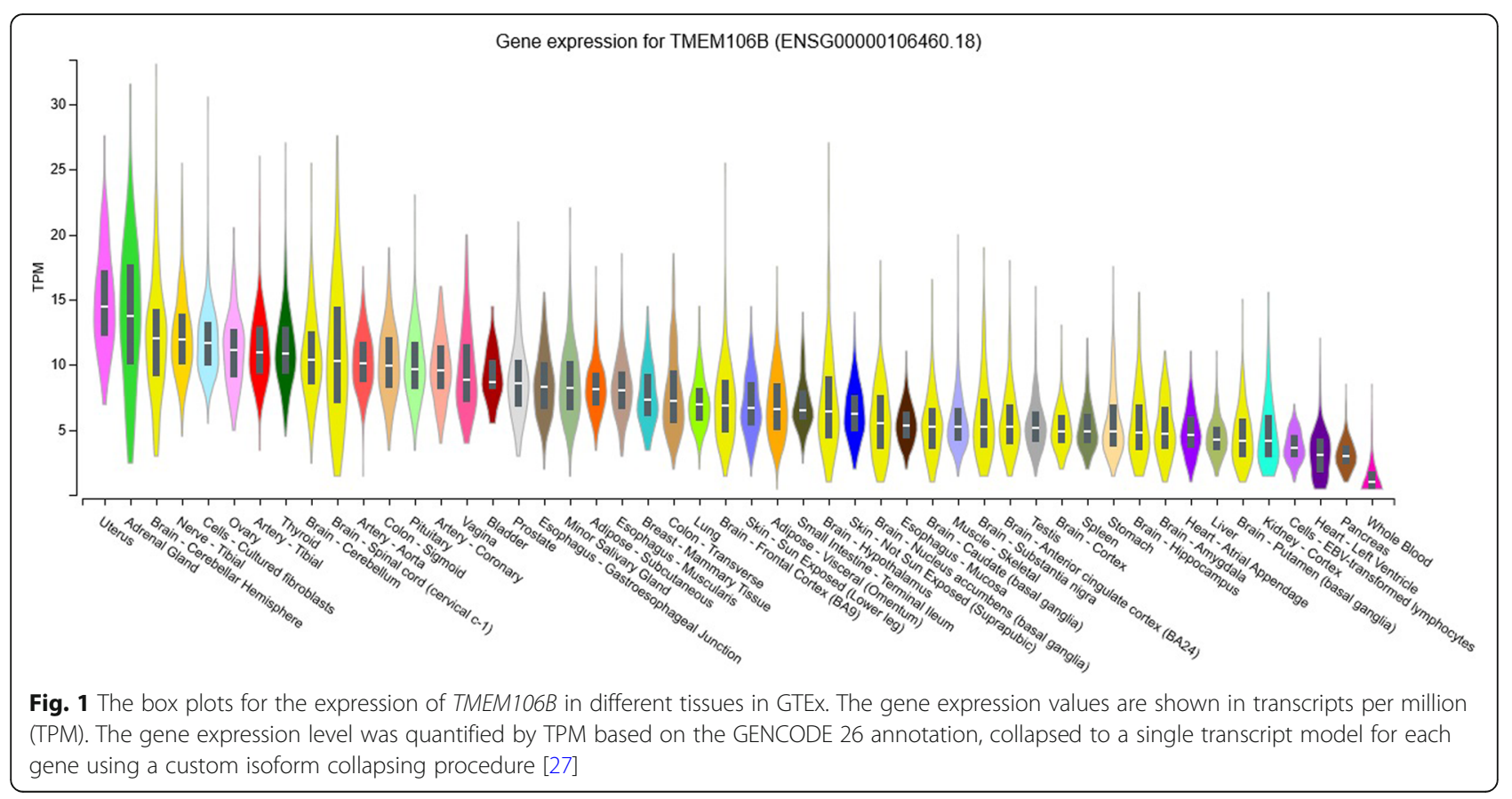


Table 3 Association between rs1990622 variant T allele and TMEM106B expression

\begin{tabular}{|c|c|c|c|c|c|}
\hline Dataset & Beta & SE & $P$ value & Brain tissue & Number \\
\hline UKBEC & 0.024 & 0.046 & $5.96 \mathrm{E}-01$ & Cerebellar cortex & 134 \\
\hline UKBEC & 0.019 & 0.053 & 7.20E-01 & Frontal cortex & 134 \\
\hline UKBEC & 0.037 & 0.054 & 4.92E-01 & Hippocampus & 134 \\
\hline UKBEC & $6.57 \mathrm{E}-05$ & 0.044 & $9.99 \mathrm{E}-01$ & Medulla & 134 \\
\hline UKBEC & 0.015 & 0.065 & $8.19 \mathrm{E}-01$ & Occipital cortex & 134 \\
\hline UKBEC & -0.003 & 0.063 & $9.63 \mathrm{E}-01$ & Putamen & 134 \\
\hline UKBEC & -0.025 & 0.058 & $6.69 E-01$ & Substantia nigra & 134 \\
\hline UKBEC & -0.030 & 0.049 & $5.38 \mathrm{E}-01$ & Temporal cortex & 134 \\
\hline UKBEC & -0.019 & 0.058 & $7.48 \mathrm{E}-01$ & Thalamus & 134 \\
\hline UKBEC & 0.046 & 0.052 & $3.78 \mathrm{E}-01$ & Intralobular white matter & 134 \\
\hline GTEx & -0.084 & 0.065 & $1.90 \mathrm{E}-01$ & Amygdala & 129 \\
\hline GTEx & -0.100 & 0.059 & $9.60 \mathrm{E}-02$ & Anterior cingulate cortex & 147 \\
\hline GTEx & -0.025 & 0.033 & $4.50 \mathrm{E}-01$ & Caudate & 194 \\
\hline GTEx & -0.130 & 0.041 & $1.50 \mathrm{E}-03$ & Cerebellar hemisphere & 175 \\
\hline GTEx & -0.150 & 0.031 & $1.90 \mathrm{E}-06$ & Cerebellum & 209 \\
\hline GTEx & -0.140 & 0.032 & $2.20 \mathrm{E}-05$ & Cortex & 205 \\
\hline GTEX & -0.089 & 0.039 & $2.20 \mathrm{E}-02$ & Frontal cortex & 175 \\
\hline GTEx & -0.009 & 0.037 & $8.20 \mathrm{E}-01$ & Hippocampus & 165 \\
\hline GTEx & -0.048 & 0.044 & $2.80 \mathrm{E}-01$ & Hypothalamus & 170 \\
\hline GTEX & 0.048 & 0.034 & $1.60 \mathrm{E}-01$ & Nucleus accumbens & 202 \\
\hline GTEX & -0.022 & 0.032 & $5.00 \mathrm{E}-01$ & Putamen & 170 \\
\hline GTEX & -0.047 & 0.062 & $4.50 \mathrm{E}-01$ & Spinal cord & 126 \\
\hline GTEx & 0.100 & 0.067 & $1.40 \mathrm{E}-01$ & Substantia nigra & 114 \\
\hline Mayo & -0.036 & - & $2.11 \mathrm{E}-01$ & Cerebellum in AD & 186 \\
\hline Mayo & 0.052 & - & $1.18 \mathrm{E}-01$ & Cerebellum in non-AD & 170 \\
\hline Mayo & -0.001 & - & $9.61 \mathrm{E}-01$ & Temporal cortex in AD & 191 \\
\hline Mayo & 0.031 & - & $1.75 E-01$ & Temporal cortex in non-AD & 181 \\
\hline
\end{tabular}

SE standard error. Beta is the regression coefficient based on the effect allele. Beta $>0$ and beta $<0$ mean that this effect allele could increase and reduce gene expression, respectively. The statistically significant association is defined to be $P<0.05 / 27=1.85 \mathrm{E}-03$. The suggestive association is defined to be $P<0.05$

cognitive deficits [31], better residual cognition [30], AD $[9,33]$, Parkinson's disease [34], and amyotrophic lateral sclerosis [34]. Importantly, Yang and colleagues identified the TMEM106B rs1990622 variant to show a significant modQTL effect, and highlighted the converging effects of $A P O E-\mathrm{A} \beta$ and TMEM106B [5]. However, it remains largely unclear about the role of rs1990622 variant in $\mathrm{AD}$. Here, we conducted comprehensive analyses including genetic association study, gene expression analysis, eQTLs analysis, and colocalization analysis.

Using the genetic association analysis, we evaluated the association of rs1990622 variant with AD using two independent large-scale GWAS datasets from IGAP and UK Biobank, and then conducted a meta-analysis [10, 11]. Interestingly, the results are consistent in both IGAP and UK Biobank, which indicated that rs1990622 was significantly associated with AD risk in both datasets (Table 2). A sex-specific analysis in UK Biobank further indicated that rs1990622 $\mathrm{T}$ allele only contributed to increased $\mathrm{AD}$ risk in females, but not in males (Table 2). Tropea and colleagues have also evaluated the association of rs1990622 variant with $\mathrm{AD}$ using $300 \mathrm{AD}$ cases and 137 neurologically normal control subjects [7]. However, Tropea and colleagues did not identify any significant association of rs1990622 with AD [7]. We think that this may be caused by inadequate sample sizes.

It is known that rs1990622 variant is a non-coding mutation. Hence, it remains unclear how rs1990622 variant affects $\mathrm{AD}$ risk. eQTLs analysis is an important method to evaluate the roles of non-coding genetic variants especially the GTEx project, which established a data resource and tissue bank to study the relationship between genetic variation and gene expression in multiple human tissues [35]. To explore the effect of rs1990622 variant in AD risk by regulating TMEM106B expression, eQTLs analysis should be conducted in 


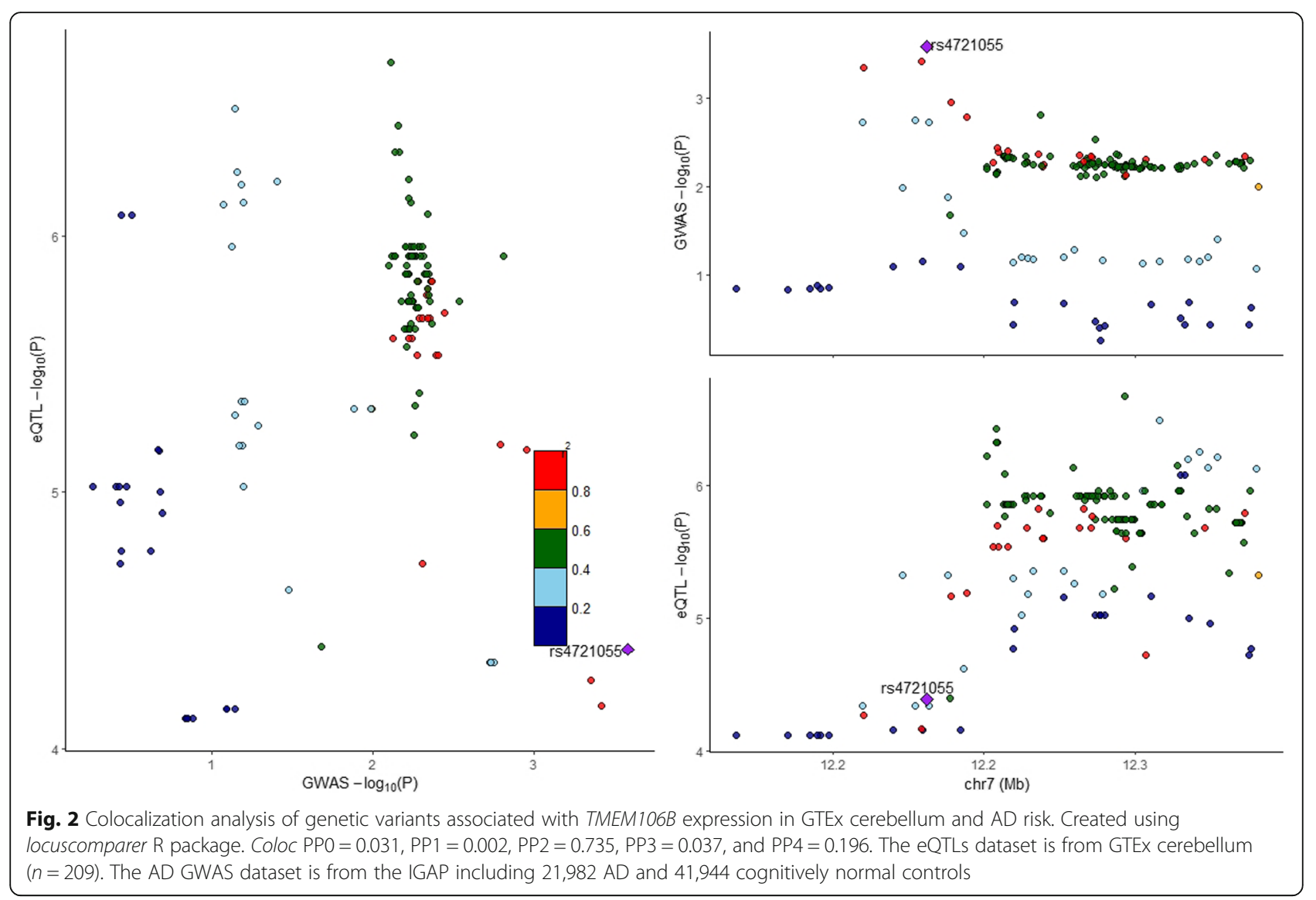

neuropathologically normal individuals or in a general population based on the three considerations. First, eQTLs analysis in AD patients could not be interpreted in terms of $\mathrm{AD}$ risk or susceptibility as lack of healthy controls or general individuals [36]. Second, it is well known that disease statuses could change the expression of a specific gene. Hence, gene expression analysis often indicated dysregulated genes in cases compared with controls [37]. Take TREM2 for example, its expression is upregulated in multiple pathological conditions such as Parkinson's disease, amyotrophic lateral sclerosis, stroke, traumatic brain injury, and $\mathrm{AD}$, compared with normal controls [37]. Until now, most eQTLs studies focusing genetic variants associated with neurological diseases were conducted in neuropathologically normal individuals, such as AD (UKBEC [38], and GSE15745 [38], and 128 normal subjects [39]), progressive supranuclear palsy (387 normal subjects) [40], schizophrenia (UKBEC [41], GTEx [41], 128 normal subjects [39], and 120 normal subjects [42]), Parkinson's disease (128 normal subjects [39], GTEx [43]), and bipolar disorder (120 normal subjects [42], GTEx [44], and UKBEC [44]). Meanwhile, other eQTLs studies using both $\mathrm{AD}$ and other controls were also reported by adjusting for disease status and some critical covariates [45, 46]. Third, Nicholson and colleagues have reviewed recent findings and found that the significant association between rs1990622 and TMEM106B mRNA expression identified in lymphoblast cells could not be successfully replicated in postmortem brain tissues [2]. It is possible that the variable levels of neuronal loss and cell type composition may have masked the association between rs1990622 and TMEM106B mRNA expression [2]. Hence, Nicholson and Rademakers suggested that eQTLs studies might be best conducted in non-diseased tissues [2].

Considering these above findings, we then performed an eQTLs analysis to evaluate the effect of rs1990622 variant on TMEM106B expression in multiple human brain tissues from neuropathologically normal individuals (UKBEC and GTEx), and further compared the findings from neurological disease individuals (Mayo and ROSMAP). In UKBEC, we found no significant association between rs1990622 variant and TMEM106B expression in 10 brain regions, as provided in Table 3. In GTEx, we found that rs1990622 T allele could significantly reduce TMEM106B expression in cerebellum $(P=1.90 \mathrm{E}-06)$, cortex $(P=2.20 \mathrm{E}-05)$, and cerebellar hemisphere $(P=1.50 \mathrm{E}-03)$. In Mayo, we found no significant association of rs1990622 with TMEM106B expression in cerebellum and temporal cortex. In 
ROSMAP, Yang and colleagues found that the rs1990622 T allele could increase TMEM106B expression in human prefrontal cortex [5]. In summary, rs1990622 variant showed different association with TMEM106B expression in neuropathologically normal individuals and neurological disease individuals (Mayo and ROSMAP). The differences were even observed across the neuropathologically normal individuals, such as UKBEC and GTEx, and across the neurological disease individuals, such as Mayo and ROSMAP.

We consider that four reasons may contribute to explain these differences. First, disease statuses may have caused these differences. Satoh and colleagues found that both the mRNA and protein levels of TMEM106B were significantly reduced in $\mathrm{AD}$ brains compared control brains [9]. Hence, the different expression of TMEM106B may further cause different eQTLs findings in AD and controls. Importantly, eQTLs or cQTL analysis using other TMEM106B variants including rs3173615 and rs1990621 further supported our findings $[4,6]$. Meanwhile, our and other studies have clearly indicated that eQTLs could vary considerably in different disease statuses [16-19, 47-52]. Second, the mean ages at death in different eQTLs datasets may have driven these differences. The mean ages at death were 55 (UKBEC), 59 (GTEx), 72 or 74 (Mayo), and 88 (ROSMAP), respectively, as provided in Table 1. Nicholson and colleagues explained that the variable levels of neuronal loss and cell type composition may have masked the association between rs1990622 and TMEM106B mRNA expression in the older population [2]. Third, the percents of females in different eQTLs datasets may have driven these differences. The percents of females were 26\% (UKBEC), 33\% (donors), 36-53\% (Mayo), and $62 \%$ (ROSMAP), respectively, as provided in Table 1. This explanation was supported by our genetic association findings that rs1990622 $\mathrm{T}$ allele only contributed to increased $\mathrm{AD}$ risk in females, but not in males (Table 2). Importantly, recent findings from GTEx also highlighted the impact of sex on gene expression across human tissues [53]. Fourth, the different descents of the selected donors may also have contributed to these differences. The donors in UKBEC, Mayo, and ROSMAP were of European descent. However, about 85.3\% donors were of European descent, and others 14.7\% were of African, Asian, and Hispanic or Latino descents, as provided in Table 1.

Using the gene expression analysis, we showed that TMEM106B had high expression in cerebellar hemisphere, tibial nerve, cerebellum, and spinal cord, but low expression in other 10 human brain tissues including frontal cortex, hypothalamus, nucleus accumbens, caudate, substantia nigra, anterior cingulate cortex, cortex, hippocampus, amygdala/amygdalae, and putamen.
Hence, these findings may explain the significant eQTLs results in cerebellum. Importantly, the colocalization analysis further provided suggestive evidence of sharing the same variant with AD risk and TMEM106B expression in cerebellum.

We also realized some limitations in our study, although these above findings. First, we only conducted a sex stratification genetic association analysis using the UK Biobank GWAS summary datasets. The sex stratification datasets in IGAP are not publicly available. Meanwhile, the original GWAS genotype datasets from IGAP and UK Biobank are not publicly available, or a long time is needed to request. Hence, we could not determine the interaction between the sex and rs1990622 genotypes using the raw data. Second, our genetic association analysis identified the female-specific role of rs1990622 in AD risk, but female- or male-specific eQTLs datasets are not publicly available. Third, we performed the eQTLs analysis to investigate the role of rs1990622 variant. In fact, the modQTL analysis may also be important, as did by Yang and colleagues. However, the original gene expression datasets limit our further modQTL analysis. Hence, we will further conduct additional sex stratification analysis, female-specific eQTLs analysis, and modQTL analysis when all these datasets are publicly available.

\section{Conclusions}

Here, we performed comprehensive analyses and found (1) FTD risk variant rs1990622 contributed to AD risk. This cross-disease approach may delineate diseasespecific and common features, which will be important for both diagnostic and therapeutic development purposes; (2) TMEM106B showed different expression in different human brain tissues especially high expression in cerebellum; (3) rs1990622 variant could regulate the expression of TMEM106B in human brain tissues, which vary considerably in different disease statuses, the mean ages at death, the percents of females, and the different descents of the selected donors; (4) colocalization analysis provided suggestive evidence that the same variant contributed to AD risk and TMEM106B expression in cerebellum. These findings highlight the importance to better understand TMEM106B function and dysfunction in the context of normal aging and neurodegenerative diseases.

\section{Abbreviations \\ AD: Alzheimer's disease; CQTL: Cell type quantitative trait loci; \\ eQTLS: Expression quantitative trait loci; GWAS: Genome-wide association} study; modQTL: Module quantitative trait loci

\section{Acknowledgements}

We thank the International Genomics of Alzheimer's Project (IGAP) and UK Biobank for the GWAS summary statistics. We thank the Braineac, Mayo Clinic, and GTEx for the eQTLs dataset resources. We also thank Marioni et al. 
for the GWAS datasets about the family history of AD. The investigators within IGAP contributed to the design and implementation of IGAP and/or provided data but did not participate in the analysis or writing of this report. IGAP was made possible by the generous participation of the control subjects, the patients, and their families. The i-Select chips was funded by the French National Foundation on AD and related disorders. EADI was supported by the LABEX (laboratory of excellence program investment for the future) DISTALZ grant, Inserm, Institut Pasteur de Lille, Université de Lille 2, and the Lille University Hospital. GERAD was supported by the Medical Research Council (grant no. 503480), Alzheimer's Research UK (grant no. 503176), the Wellcome Trust (grant no. 082604/2/07/Z), and German Federal Ministry of Education and Research (BMBF): Competence Network Dementia (CND) grant nos. 01GI0102, 01GI0711, 01GI0420. CHARGE was partly supported by the NIH/NIA grant R01 AG033193 and the NIA AG081220 and AGES contract N01-AG-12100, the NHLBI grant R01 HL105756, the Icelandic Heart Association, and the Erasmus Medical Center and Erasmus University. ADGC was supported by the NIH/NIA grants: U01 AG032984, U24 AG021886, U01 AG016976, and the Alzheimer's Association grant ADGC-10-196728. This work was partially supported by funding from the Science and Technology Beijing One Hundred Leading Talent Training Project (Z141107001514006), Beijing Municipal Administration of Hospitals' Mission Plan (SML20150802), and the National Natural Science Foundation of China (81620108011).

\section{Authors' contributions}

GYL conceived and initiated the project. YH, JYS, and GYL analyzed the data, and $Y H$, JYS, and GYL wrote the first draft of the manuscript. All authors contributed to the interpretation of the results and critical revision of the manuscript for important intellectual content and approved the final version of the manuscript.

\section{Funding}

This work was supported by funding from the National Natural Science Foundation of China (grant nos. 82071212, 61801147 and 81901181), the Mathematical Tianyuan Fund of the National Natural Science Foundation of China (grant no. 12026414), and Beijing Ten Thousand Talents Project (grant no. 2020A15). This work was also partially supported by funding from the Science and Technology Beijing One Hundred Leading Talent Training Project (Z141107001514006), the Beijing Municipal Administration of Hospitals' Mission Plan (SML20150802), and the Funds of Academic Promotion Programme of Shandong First Medical University \& Shandong Academy of Medical Sciences (no. 2019QL016, no. 2019PT007).

\section{Availability of data and materials}

All relevant data are within the paper. The authors confirm that all data underlying the findings are either fully available without restriction through consortia websites, or may be made available from consortia upon request. UK Biobank: http://www.ukbiobank.ac.uk/scientists-3/genetic-data/; https:// www.ccace.ed.ac.uk/node/335; IGAP consortium data are available at http:// web.pasteur-lille.fr/en/recherche/u744/igap/igap_download.php; https:// www.niagads.org/datasets/ng00075; eQTLs in UKBEC: http://www.braineac. org/; eQTLs in GTEx: http://www.braineac.org/; eQTLs in Mayo: https://www. niagads.org/datasets/ng00025.

\section{Ethics approval and consent to participate}

This article contains human participants collected by several studies performed by previous studies. All participants gave informed consent in all the corresponding original studies, as described in the "Methods" section. Here, our study is based on the publicly available, large-scale datasets, and not the individual-level data. Hence, ethical approval was not sought.

\section{Consent for publication}

\section{Not applicable.}

\section{Competing interests}

The authors declare no competing interests.

\section{Author details}

${ }^{1}$ School of Life Science and Technology, Harbin Institute of Technology, Harbin 150080, China. ${ }^{2}$ Shandong Provincial Hospital, Shandong First Medical University \& Shandong Academy of Medical Sciences, Jinan 250021, China. ${ }^{3}$ Department of Pathology, The Affiliated Hospital of Weifang Medical
University, Weifang 261053, China. ${ }^{4}$ Beijing Institute for Brain Disorders, Laboratory of Brain Disorders, Ministry of Science and Technology, Collaborative Innovation Center for Brain Disorders, Capital Medical University, Beijing 100069, China. ${ }^{5}$ Academy for Advanced Interdisciplinary Studies, Peking University, Beijing, China. ${ }^{6}$ Chinese Institute for Brain Research, Beijing, China. ${ }^{7}$ School of Medicine, School of Pharmaceutical Sciences, THU-PKU Center for Life Sciences, Tsinghua University, Beijing, China. ${ }^{8}$ State Key Laboratory of Medical Molecular Biology, Institute of Basic Medical Sciences, Chinese Academy of Medical Sciences, Beijing, China. ${ }^{9}$ Department of Pathophysiology, Peking Union Medical College, Beijing, China. ${ }^{10}$ Department of Anesthesiology, The Affiliated Hospital of Weifang Medical University, Weifang 261053, China. ${ }^{11}$ Key Laboratory of Cerebral Microcirculation in Universities of Shandong; Department of Neurology, Second Affiliated Hospital; Shandong First Medical University \& Shandong Academy of Medical Sciences, Taian 271000, Shandong, China. ${ }^{12}$ National Engineering Laboratory of Internet Medical Diagnosis and Treatment Technology, Xuanwu Hospital, Capital Medical University, Beijing 100053, China. ${ }^{13}$ Department of Neurology, Xuanwu Hospital, Capital Medical University, Beijing 100053, China.

Received: 13 August 2020 Accepted: 8 December 2020 Published online: 19 January 2021

\section{References}

1. Zhou X, Nicholson AM, Ren Y, Brooks M, Jiang P, Zuberi A, Phuoc HN, Perkerson RB, Matchett B, Parsons TM, et al. Loss of TMEM106B leads to myelination deficits: implications for frontotemporal dementia treatment strategies. Brain. 2020;143(6):1905-19.

2. Nicholson AM, Rademakers R. What we know about TMEM106B in neurodegeneration. Acta Neuropathol. 2016;132(5):639-51.

3. Van Deerlin VM, Sleiman PM, Martinez-Lage M, Chen-Plotkin A, Wang LS, Graff-Radford NR, Dickson DW, Rademakers R, Boeve BF, Grossman M, et al. Common variants at 7p21 are associated with frontotemporal lobar degeneration with TDP-43 inclusions. Nat Genet. 2010;42(3):234-9.

4. Li Z, Farias FHG, Dube U, Del-Aguila JL, Mihindukulasuriya KA, Fernandez MV, Ibanez L, Budde JP, Wang F, Lake AM, et al. The TMEM106B FTLDprotective variant, rs1990621, is also associated with increased neuronal proportion. Acta Neuropathol. 2020;139(1):45-61.

5. Yang HS, White CC, Klein HU, Yu L, Gaiteri C, Ma Y, Felsky D, Mostafavi S, Petyuk VA, Sperling RA, et al. Genetics of gene expression in the aging human brain reveal TDP-43 proteinopathy pathophysiology. Neuron. 2020; 107(3):496-508 e496.

6. Ren $Y$, van Blitterswijk M, Allen M, Carrasquillo MM, Reddy JS, Wang X, Beach TG, Dickson DW, Ertekin-Taner N, Asmann YW, et al. TMEM106B haplotypes have distinct gene expression patterns in aged brain. Mol Neurodegener. 2018;13(1):35.

7. Tropea TF, Mak J, Guo MH, Xie SX, Suh E, Rick J, Siderowf A, Weintraub D, Grossman M, Irwin D, et al. TMEM106B effect on cognition in Parkinson disease and frontotemporal dementia. Ann Neurol. 2019;85(6):801-11.

8. $\mathrm{Ng} \mathrm{B,} \mathrm{White} \mathrm{CC,} \mathrm{Klein} \mathrm{HU,} \mathrm{Sieberts} \mathrm{SK,} \mathrm{McCabe} \mathrm{C,} \mathrm{Patrick} \mathrm{E,} \mathrm{Xu} \mathrm{J,} \mathrm{Yu} \mathrm{L,}$ Gaiteri C, Bennett DA, et al. An XQTL map integrates the genetic architecture of the human brain's transcriptome and epigenome. Nat Neurosci. 2017;20(10):1418-26.

9. Satoh J, Kino Y, Kawana N, Yamamoto Y, Ishida T, Saito Y, Arima K. TMEM106B expression is reduced in Alzheimer's disease brains. Alzheimers Res Ther. 2014;6(2):17.

10. Kunkle BW, Grenier-Boley B, Sims R, Bis JC, Damotte V, Naj AC, Boland A, Vronskaya M, van der Lee SJ, Amlie-Wolf A, et al. Genetic meta-analysis of diagnosed Alzheimer's disease identifies new risk loci and implicates Abeta, tau, immunity and lipid processing. Nat Genet. 2019;51(3):414-30.

11. Marioni RE, Harris SE, Zhang Q, McRae AF, Hagenaars SP, Hill WD, Davies G, Ritchie CW, Gale CR, Starr JM, et al. GWAS on family history of Alzheimer's disease. Transl Psychiatry. 2018;8(1):99.

12. Ramasamy A, Trabzuni D, Guelfi S, Varghese V, Smith C, Walker R, De T, Coin L, de Silva $R$, Cookson MR, et al. Genetic variability in the regulation of gene expression in ten regions of the human brain. Nat Neurosci. 2014;17(10):1418-28.

13. Human genomics. The Genotype-Tissue Expression (GTEx) pilot analysis: multitissue gene regulation in humans. Science. 2015;348(6235):648-60.

14. Liu G, Wang T, Tian R, Hu Y, Han Z, Wang P, Zhou W, Ren P, Zong J, Jin S, et al. Alzheimer's disease risk variant rs2373115 regulates GAB2 and NARS2 expression in human brain tissues. J Mol Neurosci. 2018;66(1):37-43. 
15. Zhang Y, Wang L, Jia H, Liao M, Chen X, Xu J, Bao Y, Liu G. Genetic variants regulate $\mathrm{NR} 1 \mathrm{H} 3$ expression and contribute to multiple sclerosis risk. J Neurol Sci. 2018;390:162-5.

16. Liu G, Zhang H, Liu B, Wang T, Han Z, Ji X. rs4147929 variant minor allele increases $A B C A 7$ gene expression and $A B C A 7$ shows increased gene expression in Alzheimer's disease patients compared with controls. Acta Neuropathol. 2020;139(5):937-40.

17. Liu G, Zhang H, Liu B, Ji X. Rs2293871 regulates HTRA1 expression and affects cerebral small vessel stroke and Alzheimer's disease. Brain. 2019; 142(11):e61.

18. Liu G, Hu Y, Han Z, Jin S, Jiang Q. Genetic variant rs17185536 regulates SIM1 gene expression in human brain hypothalamus. Proc Natl Acad Sci U S A. 2019;116(9):3347-8.

19. Liu G, Jin $\mathrm{S}, \mathrm{Hu} Y$, Jiang $Q$. Disease status affects the association between rs4813620 and the expression of Alzheimer's disease susceptibility gene TRIB3. Proc Natl Acad Sci U S A. 2018;115(45):E10519-20.

20. Zou F, Chai HS, Younkin CS, Allen M, Crook J, Pankratz VS, Carrasquillo MM, Rowley CN, Nair AA, Middha S, et al. Brain expression genome-wide association study (eGWAS) identifies human disease-associated variants. PLoS Genet. 2012;8(6):e1002707.

21. Liu G, Bao X, Jiang Y, Liao M, Jiang Q, Feng R, Zhang L, Ma G, Chen Z, Wang $G$, et al. Identifying the association between Alzheimer's disease and Parkinson's disease using genome-wide association studies and proteinprotein interaction network. Mol Neurobiol. 2015;52(3):1629-36.

22. Liu G, Jiang $Y$, Chen $X$, Zhang R, Ma G, Feng R, Zhang L, Liao M, Miao Y, Chen $Z$, et al. Measles contributes to rheumatoid arthritis: evidence from pathway and network analyses of genome-wide association studies. Plos One. 2013;8(10):e75951.

23. Hu Y, Zheng L, Cheng L, Zhang Y, Bai W, Zhou W, Wang T, Han Z, Zong J, Jin S, et al. GAB2 rs2373115 variant contributes to Alzheimer's disease risk specifically in European population. J Neurol Sci. 2017;375:18-22.

24. Liu G, Sun JY, Xu M, Yang XY, Sun BL. SORL1 variants show different association with early-onset and late-onset Alzheimer's disease risk. J Alzheimers Dis. 2017;58(4):1121-8.

25. Liao M, Wang G, Quan B, Qi X, Yu Z, Feng R, Zhang L, Jiang Y, Zhang Y, Liu $G$. Analyzing large-scale samples confirms the association between rs16892766 polymorphism and colorectal cancer susceptibility. Sci Rep. 2015;5:7957.

26. Hu Y, Cheng L, Zhang Y, Bai W, Zhou W, Wang T, Han Z, Zong J, Jin S, Zhang J, et al. Rs4878104 contributes to Alzheimer's disease risk and regulates DAPK1 gene expression. Neurol Sci. 2017;38(7):1255-62.

27. Battle A, Brown CD, Engelhardt BE, Montgomery SB. Genetic effects on gene expression across human tissues. Nature. 2017;550(7675):204-13.

28. Giambartolomei C, Vukcevic D, Schadt EE, Franke L, Hingorani AD, Wallace C, Plagnol V. Bayesian test for colocalisation between pairs of genetic association studies using summary statistics. Plos Genet. 2014;10(5):e1004383.

29. Wallace $C$. Eliciting priors and relaxing the single causal variant assumption in colocalisation analyses. PLoS Genet. 2020;16(4):e1008720.

30. White CC, Yang HS, Yu L, Chibnik LB, Dawe RJ, Yang J, Klein HU, Felsky D, Ramos-Miguel A, Arfanakis K, et al. Identification of genes associated with dissociation of cognitive performance and neuropathological burden: multistep analysis of genetic, epigenetic, and transcriptional data. Plos Med. 2017;14(4):e1002287.

31. Rhinn $H$, Abeliovich A. Differential aging analysis in human cerebral cortex identifies variants in TMEM106B and GRN that regulate aging phenotypes. Cell Syst. 2017;4(4):404-15 e405.

32. Katsumata Y, Nelson PT, Ellingson SR, Fardo DW. Gene-based association study of genes linked to hippocampal sclerosis of aging neuropathology: GRN, TMEM106B, ABCC9, and KCNMB2. Neurobiol Aging. 2017;53:193 e117-25.

33. Jun $G$, Ibrahim-Verbaas CA, Vronskaya M, Lambert JC, Chung J, Naj AC, Kunkle BW, Wang LS, Bis JC, Bellenguez C, et al. A novel Alzheimer disease locus located near the gene encoding tau protein. Mol Psychiatry. 2016; 21(1):108-17.

34. Hu T, Chen Y, Ou R, Wei Q, Cao B, Zhao B, Wu Y, Song W, Chen X, Shang HF. Association analysis of polymorphisms in VMAT2 and TMEM106B genes for Parkinson's disease, amyotrophic lateral sclerosis and multiple system atrophy. J Neurol Sci. 2017;377:65-71.

35. GTEx Consortium. The GTEx Consortium atlas of genetic regulatory effects across human tissues. Science. 2020;369(6509):1318-1330.

36. Yau AC, Houtman M, Padyukov L, Holmdahl R. Reply to Liu et al.: translation of rat congenic data to humans on a conserved MHC-III haplotype associated with rheumatoid arthritis. Proc Natl Acad Sci U S A. 2016;113(42): E6323-4.

37. Gratuze M, Leyns CEG, Holtzman DM. New insights into the role of TREM2 in Alzheimer's disease. Mol Neurodegener. 2018;13(1):66.

38. Karch CM, Ezerskiy LA, Bertelsen S, Goate AM. Alzheimer's disease risk polymorphisms regulate gene expression in the ZCWPW1 and the CELF1 loci. Plos One. 2016;11(2):e0148717.

39. Blauwendraat C, Francescatto M, Gibbs JR, Jansen IE, Simon-Sanchez J, Hernandez DG, Dillman AA, Singleton AB, Cookson MR, Rizzu P, et al. Comprehensive promoter level expression quantitative trait loci analysis of the human frontal lobe. Genome Med. 2016;8(1):65.

40. Hoglinger GU, Melhem NM, Dickson DW, Sleiman PM, Wang LS, Klei L, Rademakers R, de Silva R, Litvan I, Riley DE, et al. Identification of common variants influencing risk of the tauopathy progressive supranuclear palsy. Nat Genet. 2011:43(7):699-705.

41. Bhalala OG, Nath AP, Inouye M, Sibley CR. Identification of expression quantitative trait loci associated with schizophrenia and affective disorders in normal brain tissue. Plos Genet. 2018;14(8):e1007607.

42. O'Brien HE, Hannon E, Hill MJ, Toste CC, Robertson MJ, Morgan JE, McLaughlin G, Lewis CM, Schalkwyk LC, Hall LS, et al. Expression quantitative trait loci in the developing human brain and their enrichment in neuropsychiatric disorders. Genome Biol. 2018:19(1):194.

43. Pierce S, Coetzee GA. Parkinson's disease-associated genetic variation is linked to quantitative expression of inflammatory genes. Plos One. 2017; 12(4):e0175882.

44. Akkouh IA, Skrede S, Holmgren A, Ersland KM, Hansson L, Bahrami S, Andreassen OA, Steen VM, Djurovic S, Hughes T. Exploring lithium's transcriptional mechanisms of action in bipolar disorder: a multi-step study. Neuropsychopharmacology. 2020;45(6):947-55.

45. Chung J, Zhang X, Allen M, Wang X, Ma Y, Beecham G, Montine TJ, Younkin SG, Dickson DW, Golde TE, et al. Genome-wide pleiotropy analysis of neuropathological traits related to Alzheimer's disease. Alzheimers Res Ther. 2018;10(1):22.

46. Sieberts SK, Perumal TM, Carrasquillo MM, Allen M, Reddy JS, Hoffman GE, Dang KK, Calley J, Ebert PJ, Eddy J, et al. Large eQTL meta-analysis reveals differing patterns between cerebral cortical and cerebellar brain regions. Sci Data. 2020;7(1):340.

47. Hu Y, Jin S, Cheng L, Liu G, Jiang Q. Autoimmune disease variants regulate GSDMB gene expression in human immune cells and whole blood. Proc Natl Acad Sci U S A. 2017;114(38):E7860-2.

48. Soldner F, Stelzer Y, Shivalila CS, Abraham BJ, Latourelle JC, Barrasa MI, Goldmann J, Myers RH, Young RA, Jaenisch R. Parkinson-associated risk variant in distal enhancer of alpha-synuclein modulates target gene expression. Nature. 2016:533(7601):95-9.

49. Liu G, Hu Y, Jin S, Jiang Q. Genetic variant rs763361 regulates multiple sclerosis CD226 gene expression. Proc Natl Acad Sci U S A. 2017;114(6): E906-7.

50. Liu G, Hu Y, Jin S, Zhang F, Jiang Q, Hao J. Cis-eQTLs regulate reduced LST1 gene and NCR3 gene expression and contribute to increased autoimmune disease risk. Proc Natl Acad Sci U S A. 2016;113(42):E6321-2.

51. Peters JE, Lyons PA, Lee JC, Richard AC, Fortune MD, Newcombe PJ, Richardson S, Smith KG. Insight into genotype-phenotype associations through eQTL mapping in multiple cell types in health and immunemediated disease. Plos Genet. 2016;12(3):e1005908.

52. Liu G, Hu Y, Jiang Q. Population difference and disease status affect the association between genetic variants and gene expression. Gastroenterology. 2019;157(3):894-6.

53. Oliva M, Munoz-Aguirre M, Kim-Hellmuth S, Wucher V, Gewirtz ADH, Cotter DJ, Parsana P, Kasela S, Balliu B, Vinuela A et al. The impact of sex ongene expression across human tissues. Science. 2020;369(6509):eaba3066.

\section{Publisher's Note}

Springer Nature remains neutral with regard to jurisdictional claims in published maps and institutional affiliations. 